\title{
Ex Vivo Immunomodulatory Effects of Lactobacillus-, Lacticaseibacillus-, and Bifidobacterium-Containing Synbiotics on Human Peripheral Blood Mononuclear Cells and Monocyte-Derived Dendritic Cells in the Context of Grass Pollen Allergy
}

\author{
Alexander Heldner ${ }^{1} \cdot$ Matthew D. Heath ${ }^{2} \cdot$ Benjamin Schnautz ${ }^{1} \cdot$ Sebastian $\operatorname{Kotz}^{3}$ - Adam Chaker ${ }^{1,3}$. \\ Matthias F. Kramer ${ }^{2,4}$. Constanze A. Jakwerth ${ }^{1}$ - Ulrich M. Zissler ${ }^{1}$ • Carsten B. Schmidt-Weber ${ }^{1}$. Simon Blank ${ }^{1}$ (D
}

Accepted: 18 January 2022

(c) The Author(s) 2022

\begin{abstract}
Sensing of the intestinal microbiota by the host immune system is important to induce protective immune responses. Hence, modification of the gut microbiota might be able to prevent or treat allergies, mediated by proinflammatory Th2 immune responses. The aim was to investigate the ex vivo immunomodulatory effects of the synbiotics Pollagen ${ }^{\circledR}$ and Kallergen ${ }^{\circledR}$, containing the probiotic bacterial strains Lactobacillus, Lacticaseibacillus and Bifidobacterium, in the context of grass pollen allergy. Peripheral blood mononuclear cells (PBMCs) from grass pollen-allergic patients and healthy controls were stimulated with grass pollen extract (GPE) and synbiotics and Gata3 expression and cytokine secretion analyzed. Monocyte-derived dendritic cells (MoDCs) cells were matured in the presence of GPE and synbiotics, co-cultured with autologous naïve T cells and maturation markers and cytokine secretion analyzed. GPE stimulation of PBMCs from grass pollen-allergic patients resulted in a significant higher production of the Th2 cytokines IL-4, IL-5, IL-9 and IL-13 compared to healthy controls. $\mathrm{Gata}^{+} \mathrm{CD} 4^{+} \mathrm{T}$ cell induction was independent of the allergic status. The synbiotics promoted IL-10 and IFN- $\gamma$ secretion and downregulated the GPE-induced Th2-like phenotype. Co-culturing naïve T cells with MoDCs, matured in the presence of GPE and synbiotics, shifted the GPE-induced Th2 cytokine release towards Th1-Th17-promoting conditions in allergic subjects. The investigated synbiotics are effective in downregulating the GPE-induced Th2 immune response in PBMCs from grass pollen-allergic patients as well as in autologous MoDC-T cell stimulation assays. In addition to increased IL-10 release, the data indicates a shift from a Th2- to a more Th1- and Th17-like phenotype.
\end{abstract}

Keywords Bifidobacterium $\cdot$ Grass pollen allergy $\cdot$ Immunomodulation $\cdot$ Lactobacillus $\cdot$ Lacticaseibacillus $\cdot$ Synbiotics

\section{Introduction}

Simon Blank

simon.blank@tum.de

1 Center of Allergy and Environment (ZAUM)Faculty of Medicine and Helmholtz Center MunichMember of the German Center of Lung Research (DZL), Member of the Immunology and Inflammation Initiative of the Helmholtz Association, Technical University of Munich, German Research Center for Environmental Health, Ingolstädter Landstraße 1, 85764 Munich, Germany

2 Allergy Therapeutics PLC, Worthing, UK

3 Faculty of Medicine, Department of Otolaryngology, Klinikum Rechts Der Isar, Technical University of Munich, Munich, Germany

4 Bencard Allergie GmbH, Munich, Germany
Respiratory allergies to airborne allergen sources like pollen are the most frequent type-1 hypersensitivities. In developed countries, sensitization rates to grass (Pooideae) pollen such as timothy grass (Phleum pretense) substantially increased over the last decades and nowadays range between 10 to $30 \%$ in the general population [1]. Although pollen-monitoring networks were implemented in the last few years [2], avoidance of exposure to airborne allergens is nearly impossible. Allergen-specific immunotherapy (AIT) is the only curative treatment that is able to improve allergy-driven symptoms by inducing allergen-specific immune tolerance.

Within the last few years novel therapeutic and preventive approaches were investigated. Here, modifying the 
patients' gut microbiota with probiotic and prebiotic supplements, known as synbiotic preparations, got into the focus of allergy research, prevention, and treatment [3]. Probiotics are living strains of microorganisms that have beneficial effects on the gut microbiome when administrated in adequate amounts. Prebiotics are non-digestible food ingredients that contribute to the growth and activity of beneficial bacterial strains and resident microorganisms in the gut [4]. It is thought that pro- and prebiotics are able to induce a more favorable gut colonization and strengthen immune function by immunomodulatory properties, thus contributing to the reduction of allergic symptoms as well as of the risk of allergy development in the context of allergy prevention $[5,6]$. The modulation mechanisms of probiotic bacteria are highly complex, involving a variety of effector signals, cell types, different receptors, and bacteria strains differing in their ability to trigger these effects. Besides direct affecting the host epithelial barrier function via microorganism-associated molecular patterns and pattern recognition receptors, these beneficial effects are mediated by modulation of innate immunity by influencing dendritic cell maturation. Shifting the quality of the innate immune response can lead to an increased Th1/Th2 ratio and elevated amount of regulatory T cells (Tregs) [7]. Further studies indicated that probiotic bacteria are acting as Th1/Treg inducers and may be potential candidates as adjuvants in AIT [8, 9]. It is known that an adequate maturation of the intestinal microenvironment and gut colonization in early life is crucial for protection against allergic diseases and maintaining health in adulthood [10]. Several different intestinal microbial strains and their gut colonization in early life are considered to be essential for future health [11]. After birth, fecal microbiota of neonates is dominated by Prevotella and Lactobacillus strains, then within the first weeks of life actinobacteria, mainly Bifidobacterium stains, become more dominant [12,13]. Several studies showed that breast-fed infants have more Bifidobacteria and lactobacilli and a more diverse intestinal microbial flora [12]. It is known that dysbiosis, characterized by a depletion of certain bacterial strains and altered metabolic activity, especially in childhood, is associated with an increased risk of atopy and asthma development [14]. Immunomodulatory effects of synbiotic preparations, consisting of probiotic lactic acid bacteria strains (e.g., Lactobacillus and Bifidobacterium) and prebiotic fructo-oligosaccharides (FOS), were recently studied in the context of respiratory allergies such as allergic rhinitis and asthma [15-17]. In addition, in ovalbumin-(OVA-)sensitized mice, probiotic strains were able to reduce OVA-specific IgE (sIgE) levels and airway hyper-responsiveness and, furthermore, to decrease the number of infiltrating inflammatory cells and the levels of Th2 cytokines in bronchoalveolar lavage fluid and serum [18]. Nevertheless, the results are inconclusive so far, and the efficacy of probiotics and prebiotics as potential allergy-preventive supplements has to be investigated further [19]. Hence, the aim of this study was to gain insights in the ex vivo immunomodulatory capacity of two synbiotic mixes - Pollagen ${ }^{\circledR}$ and Kallergen ${ }^{\circledR}$ - in the context of grass pollen allergy-mediated Th2-type inflammation.

\section{Methods}

\section{Patients}

Peripheral blood samples of patients with a confirmed history of grass pollen allergy and of non-grass pollen-allergic volunteers were used to isolate peripheral blood mononuclear cells (PBMCs), monocytes, and naïve $\mathrm{T}$ cells. The diagnosis of grass pollen allergy was based on a combination of clinical history and positive skin prick test and/or positive sIgE level to grass pollen extract (grass pollen extract and rPhl p1/5 (UniCAP250; Thermo Fisher Scientific, Uppsala, Sweden). Healthy volunteers were defined by a lack of allergic symptoms in their patient history and negative skin prick test results. The study was approved by the local ethics committee of the Faculty of Medicine of the Technical University of Munich (299/15 s), and all patients and volunteers gave written informed consent prior to study participation.

\section{Synbiotic Mixes}

Two commercially available synbiotic dietary supplements based on probiotics and prebiotics were tested. Pollagen ${ }^{\circledR}$ (Bencard Allergie GmbH, Munich, Germany) contains Lactobacillus acidophilus NCFM (33 billion CFU/100 g), Bifidobacterium lactis BL-04 (100 billion CFU/1006), and fructooligosaccharides (FOS) (33.33 g/100 g), and Kallergen ${ }^{\circledR}$ (Bencard Allergie $\mathrm{GmbH}$ ) contains Lacticaseibacillus rhamnosus (previously Lactobacillus rhamnosus) LR05 (38.5 billion CFU/100 g), Bifidobacterium lactis BS01 (38.5 billion CFU/100 g), and FOS $(85.5 \mathrm{~g} / 100 \mathrm{~g})$. The number of colony-forming units (CFU) of both synbiotic mixes was adjusted to the same amount of probiotic bacterial cells in each experimental setup. To obtain synbiotic culture supernatants, $1 \times 10^{6} \mathrm{CFU}$ of each synbiotic mix were cultured in $150 \mathrm{ml}$ RPMI medium (Thermo Fisher Scientific, Waltham, Massachusetts, USA) at $37^{\circ} \mathrm{C}$ starting at an $\mathrm{OD}_{600}=0.1$ until a $\mathrm{OD}_{600}=0.8$ was reached. After centrifugation (3000 rcf, $15 \mathrm{~min}$ ), culture supernatants were sterile filtered $(0.22 \mu \mathrm{m}$, Millex-GP filter unit, Merck, Darmstadt, Germany) and stored at $4{ }^{\circ} \mathrm{C}$. Concentrations of probiotic bacteria and supernatant volumes applied in the following experiments were based on their inhibitory capacity on GPE-induced Gata3 expression. In preliminary experiments, different concentrations of the bacteria and different volumes 
of the supernatants were applied to GPE-stimulated PBMCs, and the volumes with the strongest inhibitory capacity on Gata3 expression while maintaining cell proliferation and viability were selected for further experiments.

\section{Ex Vivo Stimulation of PBMCs}

PBMCs were isolated from peripheral blood of ten healthy and ten timothy grass pollen allergic patients by density gradient centrifugation (Lymphoprep, Stemcell Technologies, Vancouver, Canada) according to the manufacturers' protocol and seeded into 96-well flat-bottom plates (Nunc, Thermo Fisher Scientific) at a density of $2 \times 10^{5}$ cells per well in $200 \mu \mathrm{l}$ T cell medium (RPMI, 10\% FCS, $1 \%$ glutamine, $1 \%$ penicillin/streptomycin, $1 \%$ non-essential amino acids, $1 \%$ Na-pyruvat (all from Thermo Fisher Scientific)) with 5\% autologous serum. PBMCs were treated with $10 \mu \mathrm{g} / \mathrm{mL}$ grass pollen extract (GPE, B2-IHRP-13, Allergy Therapeutics Ltd, Worthing, United Kingdom), a pollen extract mixture from 12 temperate zone grasses, and incubated at $37{ }^{\circ} \mathrm{C}$ and $5 \%$ $\mathrm{CO}_{2}$ for 7 days. Furthermore, $2 \times 10^{5} \mathrm{CFU} / \mathrm{mL}$ of Pollagen ${ }^{\circledR}$ or Kallergen ${ }^{\circledR}$, w/o $10 \mu \mathrm{g} / \mathrm{mL}$ LPS-RS Ultrapure as TLR4 antagonist (InvivoGen, San Diego, California, USA) and $1 \mu \mathrm{l}$ Pollagen $®$ or $10 \mu \mathrm{l}$ Kallergen $®$ supernatant were added. Moreover, the same conditions were tested with the addition of $10 \mu \mathrm{g} / \mathrm{mL}$ TLR2 antibody (PAb-hTLR2, InvivoGen) for selected subjects. A total of $10 \mu \mathrm{g} / \mathrm{mL}$ phytohemagglutinin (PHA, InvivoGen) was added as positive control for PBMC stimulation. Untreated PBMCs were used as negative control. Expression of T-cell surface markers CD3 (APC-conjugated anti-human CD3, BioLegend, San Diego, California, USA), CD4 (AF700-conjugated anti-human CD4, BioLegend), CD45 (BV711-conjugated anti-human CD45, BioLegend), CD25 (BV605-conjugated anti-human CD25, BioLegend), transcription factors Gata3 (AF488-conjugated anti-human Gata3, eBioscience, San Diego, California, USA), and FoxP3 (PerCP/Cy5.5-conjugated ant-human FoxP3, eBioscience) as well as cell viability and proliferation (Zombie NIR ${ }^{\mathrm{TM}}$ Fixable Viability Kit, BioLegend; CellTrace ${ }^{\mathrm{TM}}$ CFSE Cell Proliferation Kit, Invitrogen, Carlsbad, California, USA) were measured by flow cytometry. FlowJo_V10 software (FlowJo, LLC, Ashland, Oregon, USA) was used for analysis, and gating was done with fluorescence minus one controls (FMOs) and single stains for each subject (Fig. S1). Furthermore, cytokine concentrations in cell culture supernatants were analyzed by LEGENDplex ${ }^{\mathrm{TM}}$ Human Th Panel (13-plex) assay (BioLegend) according to the manufacturer's protocol.

\section{Generation of Monocyte-Derived Dendritic Cells}

PBMCs were isolated from peripheral blood of three grass pollen-allergic patients and three healthy controls as described above, and $\mathrm{CD} 14^{+}$monocytes were isolated by magnetic cell separation (MACS, with $\mathrm{CD} 14^{+}$beads, Miltenyi Biotec, Bergisch Gladbach, Germany) and analyzed by FACS (living CD14 ${ }^{+}$cells with a purity $>95 \%$, BioLegend Zombie Aqua ${ }^{\mathrm{TM}}$ Fixable Viability Kit). For the generation of monocyte-derived dendritic cells (MoDCs) $3 \times 10^{6} \mathrm{CD} 14^{+}$ monocytes were seeded into 6-well flat-bottom cell culture plates (Falcon, Thermo Fisher Scientific) in $3 \mathrm{~mL}$ T cell medium supplemented with $50 \mathrm{U} / \mathrm{mL}$ rhIL-4/rhGM-CSF (both from Miltenyi Biotec) and incubated at $37^{\circ} \mathrm{C}$ and $5 \% \mathrm{CO}_{2}$ for 7 days. At day 4, the same amount of $\mathrm{T}$ cell medium supplemented with $50 \mathrm{U} / \mathrm{mL}$ rhIL-4/rhGM-CSF was added. After 7 days, immature MoDC (the purity of CD14- MoDCs was $>99 \%$ (data not shown)) was analyzed by FACS analysis (APC-Cy7-conjugated anti-human CD80, PE-CF594-conjugated anti-human CD86, FITC-conjugated anti-human CD209, PerCP-Cy5.5-conjugated anti-human HLA-DR, and AF700-conjugated anti-human CD14, all from BD Biosciences, San Jose, CA, USA), and viability was checked by propidium iodide staining.

\section{Stimulation and Maturation of MoDCs}

A total of $1 \times 10^{5}$ cells $/ \mathrm{mL}$ immature MoDCs were seeded into 96-well flat-bottom plates (Nunc, Thermo Fisher Scientific) and stimulated with $1 \mu \mathrm{g} / \mathrm{mL}$ lipopolysaccharides (LPS) or $1 \mu \mathrm{g} / \mathrm{mL}$ lipoteichoic acid (LTA) as positive control or $1 \mu \mathrm{g} / \mathrm{mL}$ GPE w/o $2 \times 10^{5} \mathrm{CFU} /$ $\mathrm{mL}$ Pollagen ${ }, 1 \mu \mathrm{l}$ Pollagen ${ }^{\circledR}$ supernatant, $2 \times 10^{5} \mathrm{CFU} /$ mL Kallergen ${ }^{\circledR}$, or $10 \mu \mathrm{l}$ Kallergen ${ }^{\circledR}$. Untreated MoDCs were used as negative control. After $24 \mathrm{~h}$ of maturation at $37{ }^{\circ} \mathrm{C}$ and $5 \% \mathrm{CO}_{2}$, cytokine concentrations in culture supernatants were analyzed by LEGENDplex ${ }^{\mathrm{TM}}$ Human Inflammation Panel 1 (13-plex) assay (BioLegend) according to the manufacturer's protocol. Furthermore, the expression of maturation markers CD80 (APC-Cy7conjugated anti-human CD80, BD Biosciences), CD83 (APC-conjugated anti-human, BD Biosciences), CD86 (PE-CF594-conjugated anti-human CD86, BD Biosciences), CD209 (FITC-conjugated anti-human CD209, BD Biosciences), HLA-DR (PerCP-Cy5.5-conjugated anti-human HLA-DR, BD Biosciences), CD14 (AF700conjugated anti-human CD14, BD Biosciences), and viability (Zombie NIR ${ }^{\mathrm{TM}}$ Fixable Viability Kit, BioLegend) was analyzed by flow cytometry.

\section{Co-culturing of Mature MoDCs and Naïve T Cells}

For autologous stimulation assays, the matured MoDCs were washed and co-incubated with naïve $\mathrm{T}$ cells at a ratio of 1:10 in $\mathrm{T}$ cell medium at $37{ }^{\circ} \mathrm{C}$ and $5 \% \mathrm{CO}_{2}$ for 7 days. Naïve T cells were isolated from PBMCs using the MACS naïve $\mathrm{CD} 4^{+} \mathrm{T}$ Cell Isolation Kit II (Miltenyi Biotec). A total of $20 \mathrm{U} / \mathrm{mL}$ rhIL-2 (Preprotech, Rocky Hill, New Jersey, 
USA) were added to the cells on the third day. After 7 days, cytokine concentrations in cell culture supernatants were analyzed using the LEGENDplex ${ }^{\mathrm{TM}}$ Human Th Panel (13plex) assay (BioLegend) according to the manufacturer's protocol.

\section{Statistical Analyses}

Differences between patient groups and stimulation conditions were analyzed by two-way analysis of variance (ANOVA) (GraphPad Prism, San Diego, California, USA). $P$-values of $\leq 0.05, \leq 0.01, \leq 0.001$, and $\leq 0.0001$ are shown as $*, * *, * * *$, and $* * * *$, respectively.

\section{Results}

\section{The GPE-Induced Th2-Type Response in Allergic Patients' PBMCs is Downregulated by Synbiotic Mixes}

First, the effects of GPE, synbiotic mixes, and their sterile filtered culture supernatants, containing prebiotics and probiotic metabolites, on PBMCs of grass pollen-allergic patients and healthy controls were analyzed. Stimulation of PBMCs from allergic patients with GPE resulted in a significant production of the Th2 cytokines IL-4, IL-5, IL-9, and IL-13. In healthy controls, only IL-13 was significantly upregulated upon stimulation with GPE. In contrast, in both groups, GPE induced neither IL-10 nor IFN- $\gamma$ release (Fig. 1). Stimulation with the lymphocyte-stimulating mitogen PHA served as positive control and resulted in cytokine secretion independent of the allergic status of the subjects (Fig. S2). The GPE-induced release of all Th2 cytokines was downregulated by co-culturing with both investigated synbiotic mixes, Pollagen ${ }^{\circledR}$ and Kallergen ${ }^{\circledR}$. Furthermore, both synbiotic mixes induced IL-10 and IFN- $\gamma$ release independent of the allergic status (Fig. 1). In healthy controls, stimulation with GPE led to a Th1-skewed response as demonstrated by the ratio of IFN- $\gamma$ to Th2 cytokine levels (Fig. 2). IL-17A and IL-17F are upregulated upon GPE stimulation and downregulated by addition of the synbiotic mixes in both groups (Fig. S3). Synbiotic culture supernatants were less able to downregulate the GPE-induced modulation of cytokine production and also showed no effect on IL-10 and IFN- $\gamma$ production compared to direct co-culturing with both synbiotic mixes (Fig. 1). In contrast to Th2 cytokine release, GPE-induced Gata3 expression in proliferating $\mathrm{CD} 4^{+} \mathrm{T}$ cells showed a significant upregulation in allergic subjects as well as an increased trend in non-allergic subjects. This effect was reversed by addition of both synbiotic mixes (Fig. 3). Addition of the TLR4 antagonist LPS-RS Ultrapure as well as of a TLR2 antibody partially blocked the effects of the synbiotic mixes (Figs. 1 and S4). In contrast to Gata-3,
GPE-induced expression of FoxP3 in proliferating CD4 ${ }^{+}$ $\mathrm{T}$ cells, which was higher in allergic patients compared to healthy controls, was not influenced by the addition of the synbiotic mixes (Fig. S5).

\section{Synbiotic Mixes Induce IL-10 and IL-12p70 Secretion of GPE-Matured MoDCs}

To examine how synbiotics can shift the cytokine secretion of GPE-matured MoDCs, immature MoDCs were stimulated with GPE in the presence of synbiotics or their culture supernatants. All maturation conditions led to an upregulation of the DC surface maturation markers CD83, CD86, and HLA-DR (Fig. S6). Stimulation with LPS and LTA served as positive control (Fig. S6). While maturation in the presence of GPE did not result in significant upregulation of IL-10, IL-12p70, IL-6, IL-23, and IL-1 $\beta$ secretion into culture supernatants, the addition of both synbiotic mixes enhanced production of all five cytokines independent of the allergic status of the studied subjects. This was not the case when MoDCs were matured in the presence of bacterial culture supernatants (Fig. 4). Moreover, there was a tendency toward higher IL-5 and IL-13 secretion from GPE-matured MoDCs of allergic patients (Fig. S7).

\section{Synbiotic Mixes Shift the GPE-Induced Th2 Cytokine Profile in Autologous Co-cultures of Naïve T Cells and Matured MoDCs to a More Th1- and Th17-Promoting Milieu}

Matured MoDCs were co-cultured with autologous naïve $\mathrm{T}$ cells and cytokine profiles analyzed in culture supernatants. Significant higher levels of the Th2 cytokines IL-4, IL-5, and IL-13 were detected in co-cultures of naïve T cells and GPE-matured MoDCs from grass pollen-allergic patients. Although the results showed variability within the individual patients, Pollagen ${ }^{\circledR}$ and Kallergen ${ }^{\circledR}$ were able to significantly decrease the levels of IL-5 and IL-4, respectively (Fig. 5). Intriguingly, this observation was not true for IL-9, which was upregulated only in co-cultures that contained MoDCs that were matured in the presence of GPE and synbiotic mixes, independent of the allergic status (Fig. 5). Interestingly, the synbiotic culture supernatants resulted in comparable or even more suppressive effects on IL-4, IL-5, and IL-13. Moreover, synbiotic mixes resulted in higher levels of IL-17A, IL-17F, IFN- $\gamma$, and TNF- $\alpha$ in co-culture supernatants. In the case of IFN- $\gamma$ and TNF- $\alpha$, this effect was more pronounced for grass pollen-allergic patients. Both synbiotics had only minimal effects on IL-10 levels (Fig. 5). Controls with LPS- and LTA-matured MoDCs are shown in Fig. S8. 

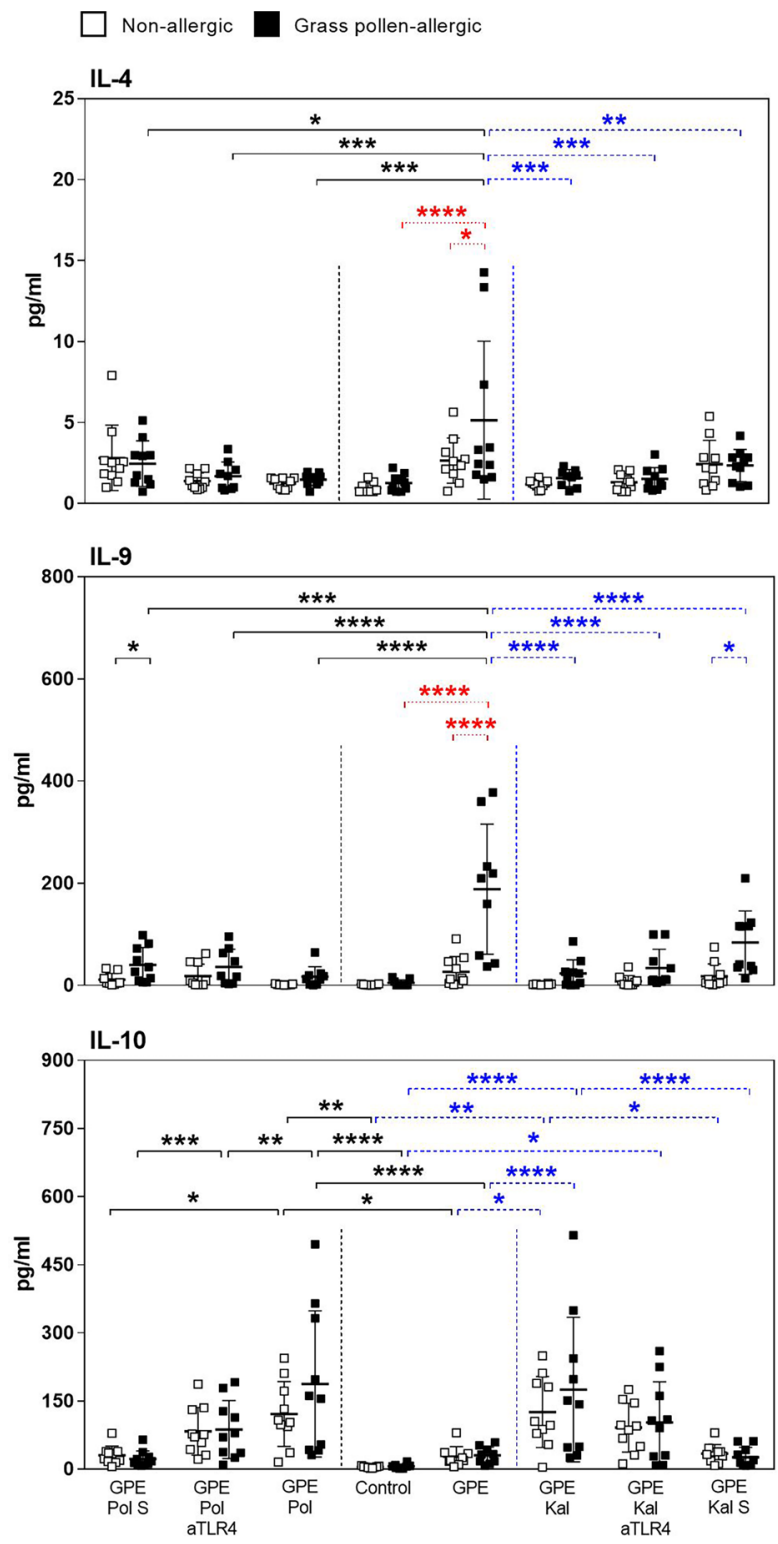

Fig. 1 Downregulation of grass pollen extract-driven Th2 cytokine production in grass pollen-allergic patients' PBMCs by synbiotic mixes. PBMCs from grass pollen-allergic patients $(n=10$; black squares) and healthy controls ( $n=10$; white squares) were stimulated with grass pollen extract (GPE). Stimulation was done w/o Pollagen (Pol)/Kallergen (Kal), LPS-RS Ultrapure (aTLR4), and Pollagen/

\section{Discussion}

In the first part of the study, the ex vivo response of PBMCs from grass pollen-allergic patients and healthy controls to GPE alone and in the additional presence of the two synbiotic mixes Pollagen ${ }^{\circledR}$ (Lactobacillus acidophilus NCFM, Bifidobacterium lactis BL-04, FOS) and Kallergen ${ }^{\circledR}$ (Lacticaseibacillus rhamnosus LR05, Bifidobacterium lactis BS01, FOS) was investigated. In fact, this study does not allow the precise contribution of each individual probiotic
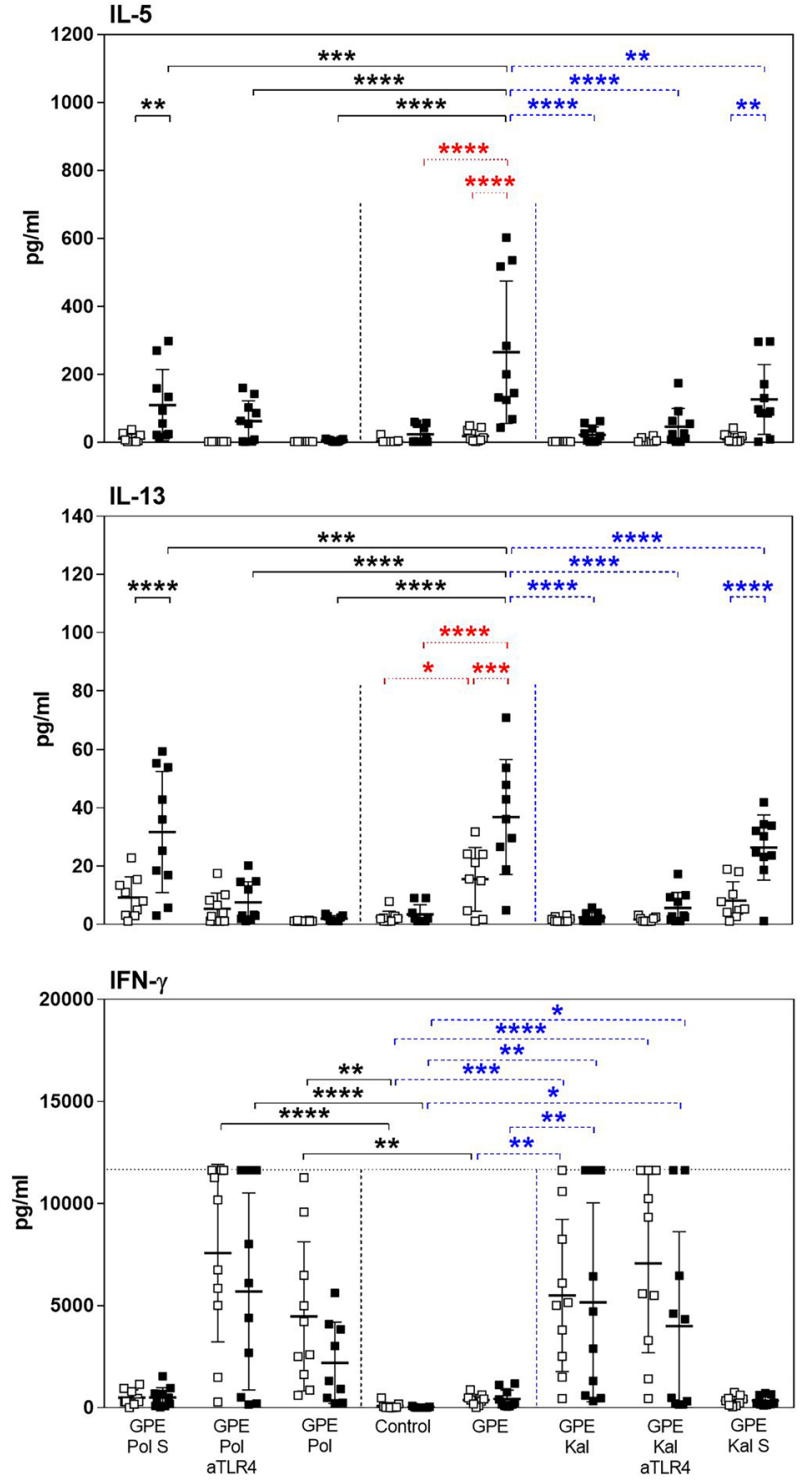

Kallergen supernatant (Pol S / Kal S). Supernatants were analyzed for the levels of IL-4, IL-5, IL-9, IL-13, IL-10, and IFN- $\gamma$. A dotted line indicates the upper detection limit for IFN- $\gamma$. Results are presented as mean \pm SD. Significant differences between controls and Pollagen, controls and Kallergen and within controls are indicated in black, blue, and red, respectively

strain to the observed effects. However, since the immunological effects of individual strains can differ greatly from mixtures of these strains [20], the detailed study of synbiotic mixes that are available for application in humans is of high interest. Hence, this study aims to provide important evidence for the evaluation if this unique combination of the synbiotics. The relatively low-quality evidence, limited comparative studies, and large heterogeneity between studies have hampered recommendations on specific probiotic formulations for the most effective management. As such, this 

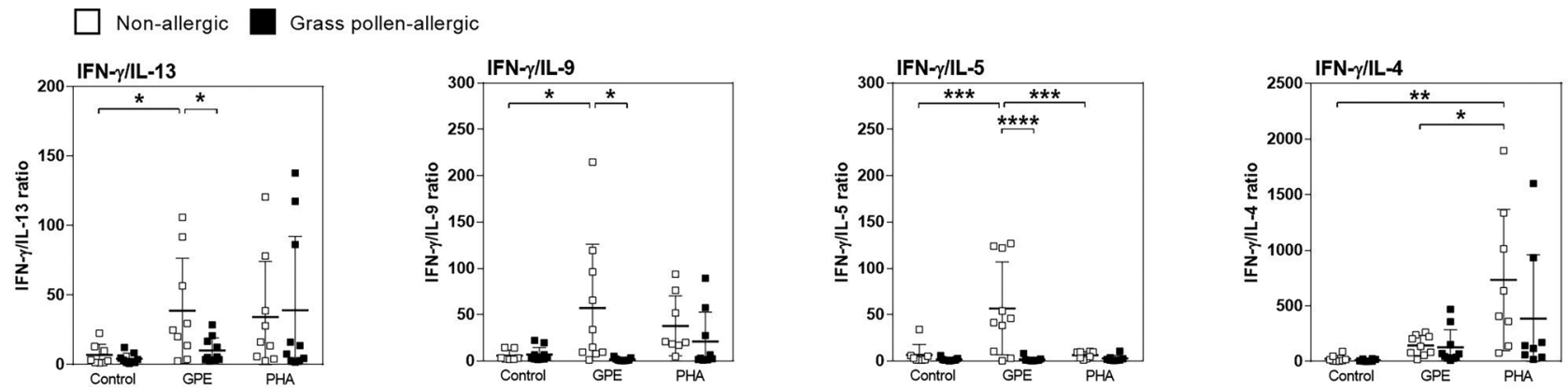

Fig. 2 Ratio of Th1 and Th2 cytokines in culture supernatants of grass pollen extract-stimulated PBMCs. PBMCs from grass pollenallergic patients ( $n=10$; black squares) and healthy controls $(n=10$; white squares) were stimulated with grass pollen extract (GPE) for

research is needed piece-by-piece to support the development of specific practical guidelines for dietary supplements containing probiotics and prebiotics.

Stimulation with GPE induced a comparable upregulation of Gata3 in proliferating $\mathrm{CD} 4^{+} \mathrm{T}$ cells independent of the allergic status of the subjects. According to other studies, GPE induced a higher Th2 cytokine release (IL-4, IL-5, IL-9, and IL-13) from PBMCs of allergic patients compared to healthy controls $[21,22]$. It was proposed that Gata3 not directly regulates IL-4 but rather acts as a chromatin-remodeling factor, to make the IL-4/IL-5 locus

7 days. Phytohemagglutinin (PHA) served as positive control. Supernatants were analyzed for the levels of IL-4, IL-5, IL-9, IL-13, and IFN-g and ratios calculated. Results are presented as mean \pm SD

accessible by interacting with other regulatory factors $[23$, 24]. The GPE-induced Th2 cytokine secretion was completely abolished by co-culturing the stimulated PBMCs with the synbiotic mixes while the addition of synbiotic culture supernatants was less effective. In all groups, the presence of probiotic bacteria seemed to induce a shift to a more Th1-like phenotype as indicated by the increased IFN- $\gamma$ levels in the culture supernatants. Furthermore, both synbiotic mixes significantly increased IL-10 secretion, which plays a central role in inflammation control by suppressing the release of pro-inflammatory cytokines [25].

Non-allergic

Grass pollen-allergic
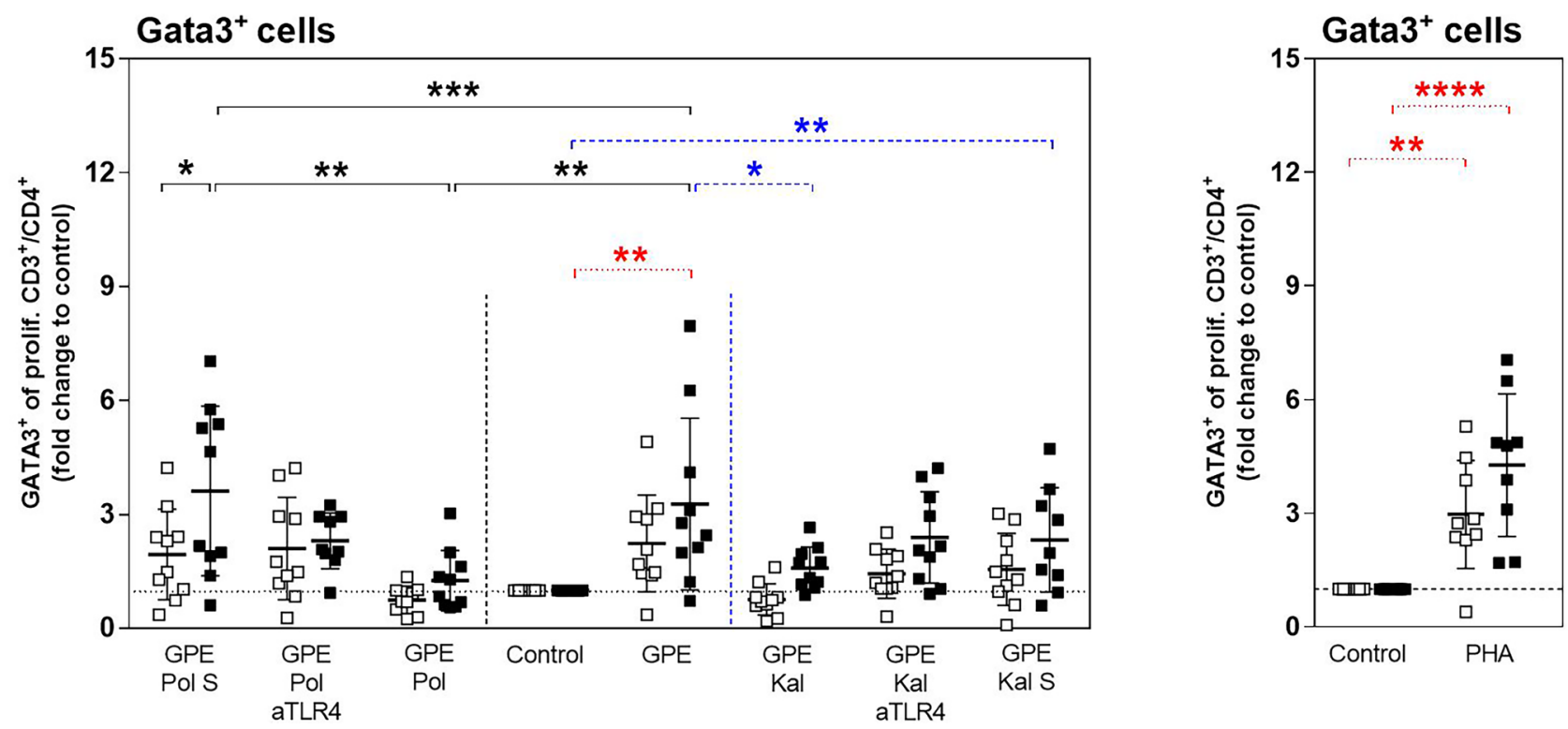

Fig. 3 Downregulation of grass pollen extract-induced Gata3 expression in PBMCs by synbiotic mixes. PBMCs from grass pollen-allergic patients ( $n=10$; black squares) and healthy controls ( $n=10$; white squares) were stimulated with grass pollen extract (GPE) for 7 days. Stimulation was done w/o Pollagen (Pol)/Kallergen (Kal), LPS-RS Ultrapure (aTLR4), and Pollagen/Kallergen supernatant (Pol S/Kal S). Phytohemagglutinin (PHA) served as positive control. Gata $3^{+}$cells of proliferating $\mathrm{CD}^{+} / \mathrm{CD}^{+}$cells were analyzed by FACS. For each patient, Gata3 expression in the negative control was set as 1, and fold change to control was calculated for each stimulation condition. Results are presented as mean \pm SD. Significant differences between controls and Pollagen, controls and Kallergen, and within controls are indicated in black, blue, and red, respectively 

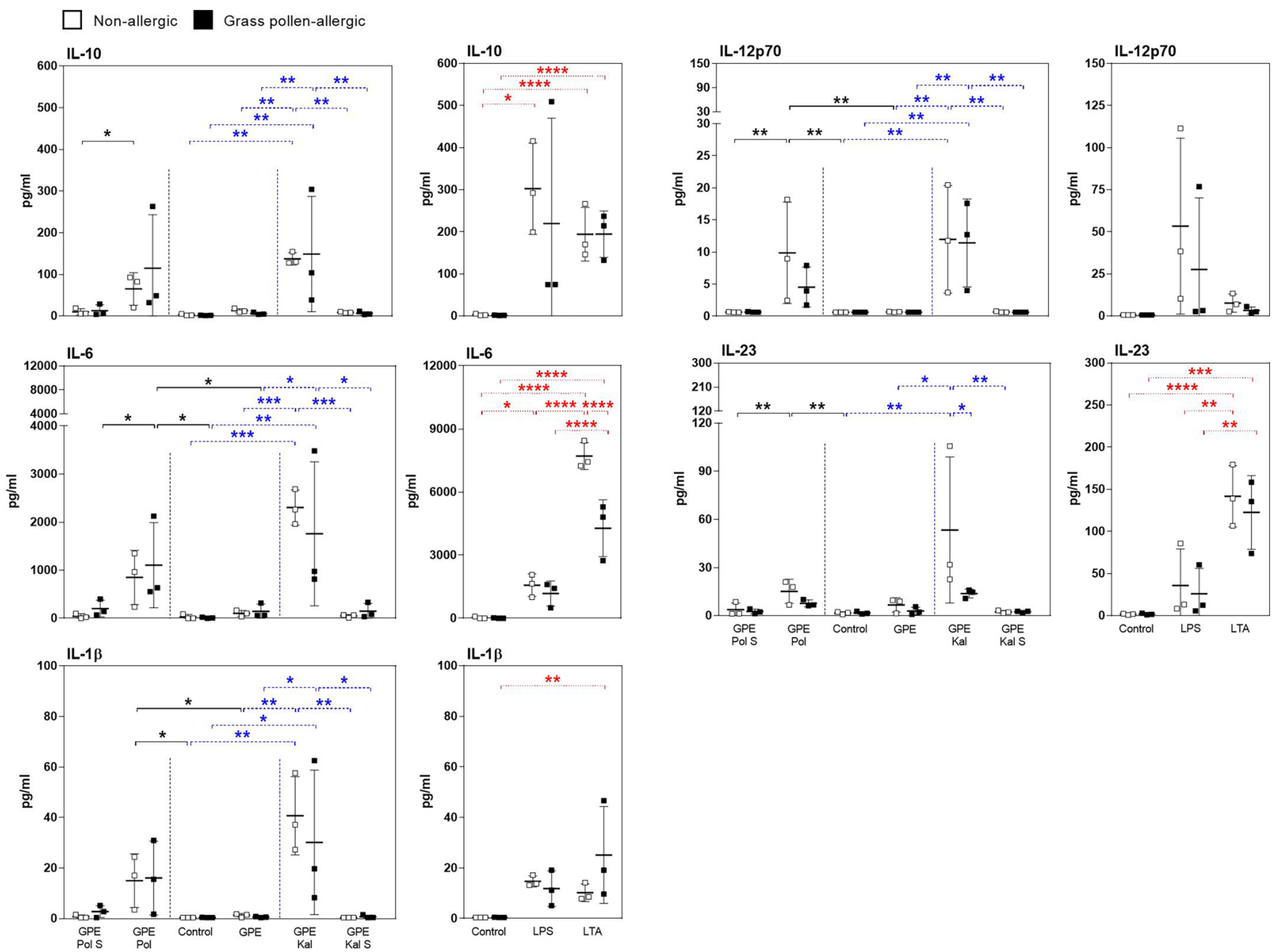

Fig. 4 Cytokine secretion of matured MoDCs. Immature MoDCs from grass pollen-allergic patients ( $n=3$; black squares) and healthy controls $(n=3$; white squares) were stimulated with grass pollen extract (GPE) for $24 \mathrm{~h}$. Stimulation was done w/o Pollagen (Pol)/ Kallergen (Kal) and Pollagen/Kallergen supernatant (Pol S/Kal S). Lipopolysaccharide (LPS) and lipoteichoic acid (LTA) served as posi-

The synbiotic mixes did not affect GPE-induced FoxP3 expression in proliferating $\mathrm{CD} 4^{+} \mathrm{T}$ cells, indicating that a quantitative shift in this $\mathrm{T}$ cell population with a potentially regulatory phenotype was not responsible for the changes observed at the cytokine level. Taken together, in this study, it could be demonstrated that GPE, which induces a Th2-like response in grass pollen-allergic patient, led to a Th1-skewed response in healthy controls as indicated by an increased ratio of secreted IFN- $\gamma$ to Th2 cytokines like IL-13, IL-9, IL-5, and IL-4. Overall, the increased Th1/Th2 cytokine ratios are higher in healthy subjects compared to grass pollen-allergic patients. The observed Th1-skewed response in healthy controls was also reported in context of a peanut allergy study [26].

Furthermore, this study investigated the involvement of TLRs in the immunomodulatory effects of the synbiotic mixes. tive controls. Supernatants were analyzed for the levels of IL-10, IL$12 p 70$, IL-6, IL-23, and IL-1 $\beta$. Results are presented as mean \pm SD. Significant differences between controls and Pollagen, controls, and Kallergen and within controls are indicated in black, blue, and red, respectively

TLRs are membrane-bound pattern recognition receptors (PRRs) which play a crucial role in innate immune responses and are expressed on immune cells, such as dendritic cells, monocytes, and epithelial cells. TLRs on host cells are essential for the recognition of conserved pathogen-associated molecular patterns (PAMPs) of microbial strains and pathogens. Signaling is followed by diverse and tightly regulated mechanisms which mediate the initiation of adaptive immune responses and release of cytokines. Blocking TLR4 as well as TLR2 signaling partially weakened the synbiotics-mediated reduction of the GPE-induced Th2 polarization in PBMCs from allergic patients. These results implicate that the immunomodulatory effects are partially mediated by TLR 4 as well as TLR 2 . In general, lipoteichoic acid of gram-positive probiotic bacteria strains is primary recognized by TLR2 and prebiotics like FOS by TLR4 $[16,27]$. The GPE used in this study was analyzed 

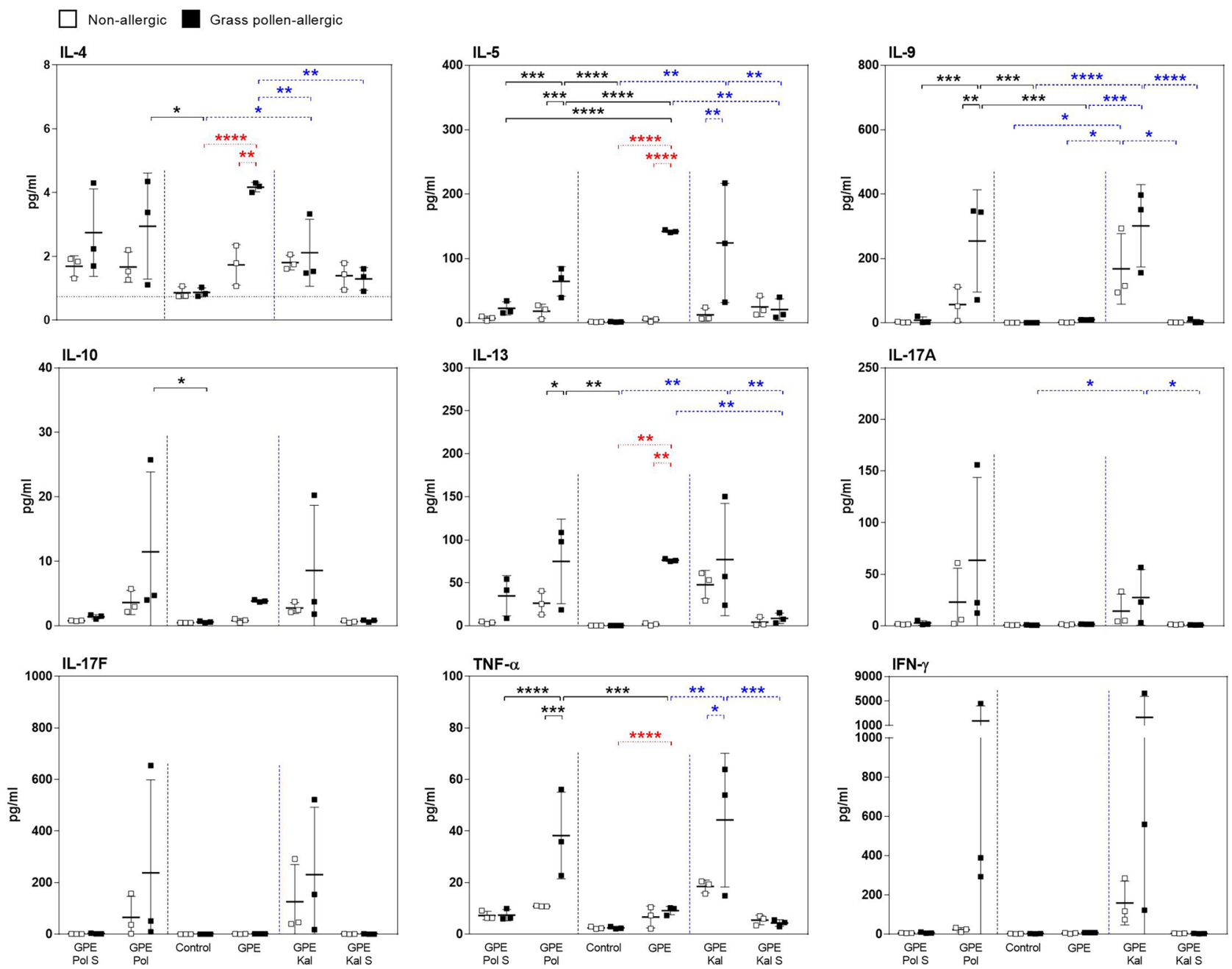

Fig. 5 Synbiotic mixes shift the grass pollen extract-(GPE)-induced Th2 cytokine profile in autologous co-cultures of naïve $T$ cells and matured MoDCs to a more Th1- and Th17-promoting milieu. Autologous naïve $\mathrm{T}$ cells from grass pollen-allergic patients $(n=3$; black squares) and healthy controls ( $n=3$; white squares) were co-cultured with GPE w/o Pollagen (Pol)/Kallergen-(Kal) or Pollagen/Kallergen

for endotoxin pyrogens, and these were within the limits stated by pharmacopeia guidance. However, a recent study showed that pollen is an important source of airborne endotoxin [28]. Therefore, an at least partial effect of LPS as a potential component of the GPE on the observed TLR4 dependence cannot be excluded. Nevertheless, activation or suppression of distinct factors of the TLR4/NFkB and other TLR pathways seems to be crucial for the probiotics-mediated modulation of inflammatory responses and are highly species- and strain-dependent [29]. Bifidobacterium lactis is able to suppress NFkB signaling in intestinal epithelial cells [30], and dietary consumption showed enhanced and improved natural immunity [31]. Probiotic mixtures containing Lactobacillus acidophilus upregulate the generation of $\mathrm{CD}^{+}{ }^{+} \mathrm{Foxp} 3^{+}$regulatory $\mathrm{T}$ cells [32] and increase IL-10 levels [33]. Lacticaseibacillus rhamnosus supernatant (Pol S/Kal S) matured MoDCs from the same donors. Supernatants were analyzed for the levels of IL-4, IL-5, IL-13, IL-10, Il-17A, IL-17F, IFN- $\gamma$, and TNF- $\alpha$. Results are presented as mean \pm SD. Significant differences between controls and Pollagen, controls and Kallergen, and within controls are indicated in black, blue, and red, respectively

species are well-studied probiotics and are able to enhance tight junction strength [34], reduce NFKB signaling and proinflammatory cytokine and chemokine release [35], and show several other immunomodulatory properties [29]. Furthermore, prebiotic supplements like non-digestible short-chain galacto- and long-chain fructo-oligosaccharides have previously showed immunomodulatory effects in the absence of probiotic strains by promoting IL-10 production, upregulation of Foxp $3^{+}$T cells, and are acting via TLR4 [16]. Interestingly, another study demonstrated that oligosaccharide preparations can be contaminated with LPS, which is responsible for some of the described immunological effects of these preparations [36], an option that cannot be fully ruled out in the current study. Nevertheless, in this ex vivo study, the immunomodulatory potential to downregulate GPE-induced Th2 immune 
response by combining two probiotic strains with prebiotic FOS, as synbiotic mixes Pollagen ${ }^{\circledR}$ and Kallergen ${ }^{\circledR}$, could be demonstrated.

To investigate the beneficial mechanisms on GPE-induced cellular immune response a bit more precise, the effects of the synbiotics on MoDCs and subsequent autologous $\mathrm{T}$ cell stimulation were analyzed. Upon activation, MoDCs undergo a maturation process and become competent in presenting antigens, which is accompanied by an upregulation of surface maturation markers like CD83, CD86, and HLA-DR. Stimulation with GPE induced comparable changes in maturation marker expression on MoDCs from allergic patients and healthy controls, as demonstrated previously by others [37]. The addition of the synbiotic mixes or synbiotic culture supernatants showed no relevant influence on surface marker expression. Both synbiotic mixes induced raised levels of IL-12p70 as well as increased IFN- $\gamma$ release by GPE-matured MoDCs. Previous reports confirmed the induction of Th1-like cytokines by Lactobacillus and Bifidobacterium strains [38-40]. Furthermore, an upregulation of IL-23, which is linked to the generation and maintenance of Th17 cells, as well as of IL-6, a key driver of IL-17 secreting $\mathrm{CD} 4^{+}$and $\mathrm{CD} 8^{+} \mathrm{T}$ cells and regulator of $T$ cell proliferation and survival [41], was demonstrated. The mechanisms responsible for Th2 polarization are still not fully understood, but it was shown that early IL-4 production in absence of IL-12 induces Th2 differentiation [42]. In this study, a tendency toward elevated release of Th2 cytokines like IL-4, IL-5, and IL-13 in GPE-stimulated MoDCs from grass pollen-allergic patients compared to healthy controls could be demonstrated. Together, these findings indicate a polarization toward Th1- and Th17-promoting conditions, by adding synbiotics to GPE during maturation of MoDCs. The sole addition of synbiotic culture supernatants did not lead to this significant shift in cytokine secretion, suggesting that the amount or nature of bacterial products released during culturing of the probiotic bacteria was not sufficient to induce the same effects. One limitation of this study, which should be addressed in future analyses, is that the composition and nature of the immunomodulatory metabolites in the synbiotic culture supernatants are not known. Besides short-chain fatty acids that can modulate epithelial integrity and the anti-inflammatory immune response, a variety of other immunomodulatory prebiotic and probiotic metabolites and other secreted molecules have been described. These include secreted protein p40 (barrier function, cell survival, mucin, and IgA production), polysaccharide A (IL-10 production, Treg induction), peptidoglycan (IL10 production, Treg induction), lipoteichoic acid (barrier function, protective immune response), exopolysaccharides (anti-inflammatory), indole-3-aldehdye (immune responses for epithelial cell protection), and others [43, 44]. Furthermore, both synbiotics were able to induce anti-inflammatory
IL-10 release. Both DCs and Tregs are relevant sources of IL-10 [45]. Irrespective of the allergic status of the individuals examined, enhanced IL-10 production by MoDCs matured in the presence of GPE and the synbiotic mixes was observed, which hints to a relevant role of these cells as IL-10 source after contact with the synbiotics. In comparison, IL-10 secretion was very low in supernatants of $\mathrm{T}$ cells co-cultured with MoDCs that had matured in the presence of GPE and synbiotic mixes. However, the source of secreted IL-10 in this experimental setup remains elusive. IL-10- and IL-12p70-producing tolerogenic MoDCs are able to induce Treg development $[16,46]$ and are known to suppress allergen-specific Th2 responses [47]. The balance between the anti-inflammatory cytokine IL-10 and proinflammatory cytokine IL-12 is important for host immunity, and the modulation mechanisms by different probiotic Lactobacillus and Bifidobacterium strains are not yet fully understood [38].

Next, cytokine secretion of co-cultures of matured MoDCs with autologous naïve $\mathrm{T}$ cells of grass pollen-allergic patients and healthy controls was analyzed. Th2 cytokine levels (IL-4, IL-5, and IL-13) were significantly higher in supernatants of GPE-matured MoDC-T cell-co-cultures of grass pollen-allergic patients compared to healthy controls. It was demonstrated previously by others that $\mathrm{Th} 2$ cytokine production in autologous stimulation assays of allergic patients could be clearly distinguished from healthy individuals $[48,49]$. MoDCs matured in the presence of GPE together with the synbiotic mixes, or synbiotic culture supernatants induced a less Th2-like cytokine response in coculture with naïve $\mathrm{T}$ cells. Additionally, the synbiotics increased the levels of IFN- $\gamma$, TNF- $\alpha$, IL-17A, and IL-17F again indicating a shift toward Th1- and Th17-promoting conditions.

Taken together, these results provide evidence that the synbiotic mixes Pollagen $₫$ and Kallergen $®$ are efficient in downregulating the GPE-induced Th2 immune response in PBMCs from grass pollen-allergic patients as well as in autologous MoDC-T cell-stimulation assays ex vivo. In addition to elevated IL-10 secretion, the data indicates a shift from a Th2- to a more Th1- and Th17-like phenotype. Although the presented study has limitations, like experimental models for a complex in vivo situation, we still believe that our ex vivo experiments can mimic the immunological events in the mucosa-associated lymphoid tissue (MALT), where intestinal lymphocytes come into contact with synbiotic mixes. MALT structures are the main sources of IgA-producing plasma cells along the surfaces of all mucosal tissues. These structures include $\mathrm{M}$ cells, which serve as entry sites for the luminal antigen to induce efficient immune responses [50]. Morphological features of $\mathrm{M}$ cells reveal a lack of microvilli but show basolateral pockets, where mononuclear phagocytes and lymphocytes are located in close contact with each other and the $\mathrm{M}$ cells and where antigen encounter takes place. The $\mathrm{M}$ cells however 
can be reduced to their role as sheath and particle transfer station. In addition, DCs are constantly sampling the luminal content of the intestine to monitor it for pathogens [51], an interaction that partially is mimicked in the experiments, in which MoDCs matured in the presence of synbiotics were co-cultured with autologous naïve $\mathrm{T}$ cells. The present study thereby provides first insights into the immunomodulatory properties of the investigated synbiotic mixes in the context of grass pollen allergy and suggests potential administration of dietary supplements for reduction or even prevention of allergic symptoms.

Supplementary Information The online version contains supplementary material available at https://doi.org/10.1007/s12602-022-09920-w.

Author Contribution Alexander Heldner performed the experiments, analyzed the data, and wrote the manuscript. Matthew D. Heath critically revised the final version of the manuscript. Benjamin Schnautz contributed to the analysis of data. Sebastian Kotz coordinated the recruitment of allergic patients and healthy controls and collected and analyzed the data. Adam Chaker coordinated the recruitment of allergic patients and healthy controls; collected, analyzed, and discussed the data; and critically revised the final version of the manuscript. Matthias F. Kramer discussed the data and critically revised the final version of the manuscript. Constanze A. Jakwerth and Ulrich M. Zissler discussed the data and contributed to the revised version of the manuscript. Carsten B. SchmidtWeber initiated and supervised the study, contributed to the interpretation of the data, and critically revised the manuscript. Simon Blank initiated and supervised the study, analyzed the data, and wrote the manuscript.

Funding Open Access funding enabled and organized by Projekt DEAL. This study was financed in part by Bencard Allergie $\mathrm{GmbH}$ to Simon Blank. The company had no influence on the study design, the collection, analysis and interpretation of data or the decision to submit the article for publication. Moreover, this work was supported by the Helmholtz Association, Future Topic "Immunology and Inflammation" (ZT-0027) to Simon Blank and Carsten B. Schmidt-Weber.

Availability of Data and Material All data is included in the text; however, the raw data of this article will be made available by the authors, without undue reservation, to any qualified researcher.

\section{Declarations}

Ethics Approval and Consent to Participate The study was approved by the local ethics committee of the Faculty of Medicine of the Technical University of Munich (299/15 s), and all the patients and volunteers gave written informed consent prior to study participation. The study was performed in accordance with the ethical standards as laid down in the 1964 Declaration of Helsinki and its later amendments or comparable ethical standards.

Consent for Publication Not applicable.

Competing Interests Simon Blank reports grants and personal fees from Bencard Allergie $\mathrm{GmbH}$, grants and personal fees from Thermo Fisher Scientific, and grants from Allergy Therapeutics, outside the submitted work. Adam Chaker reports grants and other from Allergopharma, from ALK Abello, Bencard/Allergy Therapeutics, ASIT Biotech, Lofarma, GSK, Novartis, LETI, Roche, Zeller, Sanofi Genzyme/ Regeneron, European Institute of Technology, AstraZeneca, and Immu- notek, all outside the submitted work. Matthew D. Heath and Matthias F. Kramer are employees of Allergy Therapeutics plc/Bencard Allergie $\mathrm{GmbH}$, which supported this work. Further, Allergy Therapeutics plc/ Bencard Allergie $\mathrm{GmbH}$ is a manufacturer of allergen immunotherapy and synbiotic products. Carsten B. Schmidt-Weber reports personal fees from Bencard and personal fees from Allergopharma, outside the submitted work. The other authors declare no competing interests.

Open Access This article is licensed under a Creative Commons Attribution 4.0 International License, which permits use, sharing, adaptation, distribution and reproduction in any medium or format, as long as you give appropriate credit to the original author(s) and the source, provide a link to the Creative Commons licence, and indicate if changes were made. The images or other third party material in this article are included in the article's Creative Commons licence, unless indicated otherwise in a credit line to the material. If material is not included in the article's Creative Commons licence and your intended use is not permitted by statutory regulation or exceeds the permitted use, you will need to obtain permission directly from the copyright holder. To view a copy of this licence, visit http://creativecommons.org/licenses/by/4.0/.

\section{References}

1. Akdis CA, Agache I (2014) Global Atlas of Allergy. European Academy of Allergy and Clinical Immunology. Zurich

2. Buters JTM, Antunes C, Galveias A, Bergmann KC, Thibaudon M, Galan C, Schmidt-Weber C, Oteros J (2018) Pollen and spore monitoring in the world. Clin Transl Allergy 8:9. https://doi.org/ 10.1186/s13601-018-0197-8

3. Azad MAK, Sarker M, Li T, Yin J (2018) Probiotic species in the modulation of gut microbiota: an overview. Biomed Res Int 2018:9478630. https://doi.org/10.1155/2018/9478630

4. Gibson GR, Hutkins R, Sanders ME, Prescott SL, Reimer RA, Salminen SJ, Scott K, Stanton C, Swanson KS, Cani PD, Verbeke K, Reid G (2017) Expert consensus document: The International Scientific Association for Probiotics and Prebiotics (ISAPP) consensus statement on the definition and scope of prebiotics. Nat Rev Gastroenterol Hepatol 14(8):491-502. https://doi.org/10. 1038/nrgastro.2017.75

5. Kalliomaki M, Antoine JM, Herz U, Rijkers GT, Wells JM, Mercenier A (2010) Guidance for substantiating the evidence for beneficial effects of probiotics: prevention and management of allergic diseases by probiotics. J Nutr 140(3):713S-S721. https://doi.org/10.3945/jn.109. 113761

6. West CE, Dzidic M, Prescott SL, Jenmalm MC (2017) Bugging allergy; role of pre-, pro- and synbiotics in allergy prevention. Allergol Int 66(4):529-538. https://doi.org/10.1016/j.alit.2017.08. 001

7. Mennini M, Dahdah L, Artesani MC, Fiocchi A, Martelli A (2017) Probiotics in asthma and allergy prevention. Front Pediatr 5:165. https://doi.org/10.3389/fped.2017.00165

8. Fassio F, Guagnini F (2018) House dust mite-related respiratory allergies and probiotics: a narrative review. Clin Mol Allergy 16:15. https://doi.org/10.1186/s12948-018-0092-9

9. Van Overtvelt L, Moussu H, Horiot S, Samson S, Lombardi V, Mascarell L, van de Moer A, Bourdet-Sicard R, Moingeon P (2010) Lactic acid bacteria as adjuvants for sublingual allergy vaccines. Vaccine 28(17):2986-2992. https://doi.org/10.1016/j. vaccine.2010.02.009

10. Martin R, Nauta AJ, Ben Amor K, Knippels LM, Knol J, Garssen J (2010) Early life: gut microbiota and immune development in infancy. Benef Microbes 1(4):367-382. https://doi.org/10.3920/ BM2010.0027 
11. Zhuang L, Chen H, Zhang S, Zhuang J, Li Q, Feng Z (2019) Intestinal microbiota in early life and its implications on childhood health. Genomics Proteomics Bioinformatics 17(1):13-25. https:// doi.org/10.1016/j.gpb.2018.10.002

12. Dominguez-Bello MG, Costello EK, Contreras M, Magris M, Hidalgo G, Fierer N, Knight R (2010) Delivery mode shapes the acquisition and structure of the initial microbiota across multiple body habitats in newborns. Proc Natl Acad Sci U S A 107(26):11971-11975. https://doi.org/10.1073/pnas.1002601107

13. Hill CJ, Lynch DB, Murphy K, Ulaszewska M, Jeffery IB, O'Shea CA, Watkins C, Dempsey E, Mattivi F, Tuohy K, Ross RP, Ryan CA, PW OT, Stanton C (2017) Evolution of gut microbiota composition from birth to 24 weeks in the INFANTMET Cohort. Microbiome 5(1):4. https://doi.org/10.1186/s40168-016-0213-y

14. Fujimura KE, Sitarik AR, Havstad S, Lin DL, Levan S, Fadrosh D, Panzer AR, LaMere B, Rackaityte E, Lukacs NW, Wegienka G, Boushey HA, Ownby DR, Zoratti EM, Levin AM, Johnson CC, Lynch SV (2016) Neonatal gut microbiota associates with childhood multisensitized atopy and $\mathrm{T}$ cell differentiation. Nat Med 22(10):1187-1191. https://doi.org/10.1038/nm.4176

15. Ouwehand AC, Nermes M, Collado MC, Rautonen N, Salminen S, Isolauri E (2009) Specific probiotics alleviate allergic rhinitis during the birch pollen season. World J Gastroenterol 15(26):32613268. https://doi.org/10.3748/wjg.15.3261

16. Lehmann S, Hiller J, van Bergenhenegouwen J, Knippels LM, Garssen J, Traidl-Hoffmann C (2015) In vitro evidence for immune-modulatory properties of non-digestible oligosaccharides: direct effect on human monocyte derived dendritic cells. PLoS One 10(7):e0132304. https://doi.org/10.1371/journal.pone. 0132304

17. Zuccotti G, Meneghin F, Aceti A, Barone G, Callegari ML, Di Mauro A, Fantini MP, Gori D, Indrio F, Maggio L, Morelli L, Corvaglia L, Italian Society of N (2015) Probiotics for prevention of atopic diseases in infants: systematic review and meta-analysis. Allergy 70(11):1356-1371. https://doi.org/10.1111/all.12700

18. Wu CT, Chen PJ, Lee YT, Ko JL, Lue KH (2016) Effects of immunomodulatory supplementation with Lactobacillus rhamnosus on airway inflammation in a mouse asthma model. J Microbiol Immunol Infect 49(5):625-635. https://doi.org/10.1016/j.jmii. 2014.08.001

19. Forsberg A, West CE, Prescott SL, Jenmalm MC (2016) Pre- and probiotics for allergy prevention: time to revisit recommendations? Clin Exp Allergy 46(12):1506-1521. https://doi.org/10. 1111/cea.12838

20. Lavasani S, Dzhambazov B, Nouri M, Fak F, Buske S, Molin G, Thorlacius H, Alenfall J, Jeppsson B, Westrom B (2010) A novel probiotic mixture exerts a therapeutic effect on experimental autoimmune encephalomyelitis mediated by IL-10 producing regulatory T cells. PLoS One 5(2):e9009. https://doi.org/10.1371/ journal.pone.0009009

21. Van Hemelen D, Oude Elberink JN, Bohle B, Heimweg J, Nawijn MC, van Oosterhout AJ (2011) Flow cytometric analysis of cytokine expression in short-term allergen-stimulated $\mathrm{T}$ cells mirrors the phenotype of proliferating $\mathrm{T}$ cells in long-term cultures. J Immunol Methods 371(1-2):114-121. https://doi.org/10.1016/j. jim.2011.06.019

22. Kahler H, Stuwe H, Cromwell O, Fiebig H (1999) Reactivity of T cells with grass pollen allergen extract and allergoid. Int Arch Allergy Immunol 120(2):146-157. https://doi.org/10.1159/000024233

23. Glimcher LH, Singh H (1999) Transcription factors in lymphocyte development - T and B cells get together. Cell 96(1):13-23. https://doi.org/10.1016/s0092-8674(00)80955-1

24. Hwang SS, Lee S, Lee W, Lee GR (2010) GATA-binding protein-3 regulates $\mathrm{T}$ helper type 2 cytokine and ifng loci through interaction with metastasis-associated protein 2. Immunology 131(1):50-58. https://doi.org/10.1111/j.1365-2567.2010.03271.x
25. Coomes SM, Kannan Y, Pelly VS, Entwistle LJ, Guidi R, PerezLloret J, Nikolov N, Muller W, Wilson MS (2017) CD4(+) Th2 cells are directly regulated by IL-10 during allergic airway inflammation. Mucosal Immunol 10(1):150-161. https://doi.org/10. 1038/mi.2016.47

26. Turcanu V, Maleki SJ, Lack G (2003) Characterization of lymphocyte responses to peanuts in normal children, peanut-allergic children, and allergic children who acquired tolerance to peanuts. J Clin Invest 111(7):1065-1072. https://doi.org/10.1172/ JCI16142

27. Laino J, Villena J, Kanmani P, Kitazawa H (2016) Immunoregulatory effects triggered by lactic acid bacteria exopolysaccharides: new insights into molecular interactions with host cells. Microorganisms 4(3). https://doi.org/10.3390/microorganisms4030027

28. Oteros J, Bartusel E, Alessandrini F, Nunez A, Moreno DA, Behrendt H, Schmidt-Weber C, Traidl-Hoffmann C, Buters J (2019) Artemisia pollen is the main vector for airborne endotoxin. J Allergy Clin Immunol 143(1):369-377 e5. https://doi.org/10. 1016/j.jaci.2018.05.040

29. Llewellyn A, Foey A (2017) Probiotic modulation of innate cell pathogen sensing and signaling events. Nutrients 9(10). https:// doi.org/10.3390/nu9101156

30. Kim SW, Kim HM, Yang KM, Kim SA, Kim SK, An MJ, Park JJ, Lee SK, Kim TI, Kim WH, Cheon JH (2010) Bifidobacterium lactis inhibits NF-kappaB in intestinal epithelial cells and prevents acute colitis and colitis-associated colon cancer in mice. Inflamm Bowel Dis 16(9):1514-1525. https://doi.org/10.1002/ibd.21262

31. Arunachalam K, Gill HS, Chandra RK (2000) Enhancement of natural immune function by dietary consumption of Bifidobacterium lactis (HN019). Eur J Clin Nutr 54(3):263-267. https://doi. org/10.1038/sj.ejen.1600938

32. Kwon HK, Lee CG, So JS, Chae CS, Hwang JS, Sahoo A, Nam $\mathrm{JH}$, Rhee JH, Hwang KC, Im SH (2010) Generation of regulatory dendritic cells and CD4(+)Foxp3(+) T cells by probiotics administration suppresses immune disorders. Proc Natl Acad Sci USA 107(5):2159-2164. https://doi.org/10.1073/pnas.0904055107

33. Zeuthen LH, Fink LN, Frokiaer H (2008) Toll-like receptor 2 and nucleotide-binding oligomerization domain-2 play divergent roles in the recognition of gut-derived lactobacilli and bifidobacteria in dendritic cells. Immunology 124(4):489-502. https://doi.org/10. $1111 / \mathrm{j} .1365-2567.2007 .02800 . x$

34. Orlando A, Linsalata M, Notarnicola M, Tutino V, Russo F (2014) Lactobacillus GG restoration of the gliadin induced epithelial barrier disruption: the role of cellular polyamines. BMC Microbiol 14:19. https://doi.org/10.1186/1471-2180-14-19

35. Bermudez-Brito M, Munoz-Quezada S, Gomez-Llorente C, Romero F, Gil A (2014) Lactobacillus rhamnosus and its cell-free culture supernatant differentially modulate inflammatory biomarkers in Escherichia coli-challenged human dendritic cells. Br J Nutr 111(10):1727-1737. https://doi.org/10.1017/S0007114513004303

36. Perdijk O, van Neerven RJJ, Meijer B, Savelkoul HFJ, Brugman S (2018) Induction of human tolerogenic dendritic cells by 3'-sialyllactose via TLR4 is explained by LPS contamination. Glycobiology 28(3):126-130. https://doi.org/10.1093/glycob/cwx106

37. Bellinghausen I, Brand U, Knop J, Saloga J (2000) Comparison of allergen-stimulated dendritic cells from atopic and nonatopic donors dissecting their effect on autologous naive and memory $\mathrm{T}$ helper cells of such donors. J Allergy Clin Immunol 105(5):988996. https://doi.org/10.1067/mai.2000.105526

38. Morita H, He F, Fuse T, Ouwehand AC, Hashimoto H, Hosoda M, Mizumachi K, Kurisaki J (2002) Cytokine production by the murine macrophage cell line $\mathrm{J} 7741$ after exposure to lactobacilli. Biosci Biotechnol Biochem 66(9):1963-6. https://doi.org/10. 1271/bbb.66.1963

39. Kato I, Tanaka K, Yokokura T (1999) Lactic acid bacterium potently induces the production of interleukin-12 and interferon-gamma by 
mouse splenocytes. Int J Immunopharmacol 21(2):121-131. https:// doi.org/10.1016/s0192-0561(98)00072-1

40. Foligne B, Nutten S, Grangette C, Dennin V, Goudercourt D, Poiret S, Dewulf J, Brassart D, Mercenier A, Pot B (2007) Correlation between in vitro and in vivo immunomodulatory properties of lactic acid bacteria. World J Gastroenterol 13(2):236-243. https://doi.org/10.3748/wjg.v13.i2.236

41. Hunter CA, Jones SA (2015) IL-6 as a keystone cytokine in health and disease. Nat Immunol 16(5):448-457. https://doi.org/10.1038/ni.3153

42. Seder RA, Paul WE (1994) Acquisition of lymphokine-producing phenotype by CD4+ T cells. Annu Rev Immunol 12:635-673. https://doi.org/10.1146/annurev.iy.12.040194.003223

43. Liu Q, Yu Z, Tian F, Zhao J, Zhang H, Zhai Q, Chen W (2020) Surface components and metabolites of probiotics for regulation of intestinal epithelial barrier. Microb Cell Fact 19(1):23. https:// doi.org/10.1186/s12934-020-1289-4

44. Yan F, Polk DB (2020) Probiotics and probiotic-derived functional factors-mechanistic insights into applications for intestinal homeostasis. Front Immunol 11:1428. https://doi.org/10.3389/ fimmu. 2020.01428

45. Couper KN, Blount DG, Riley EM (2008) IL-10: the master regulator of immunity to infection. J Immunol 180(9):5771-5777. https://doi.org/10.4049/jimmunol.180.9.5771

46. Smits HH, Engering A, van der Kleij D, de Jong EC, Schipper K, van Capel TM, Zaat BA, Yazdanbakhsh M, Wierenga EA, van Kooyk Y, Kapsenberg ML (2005) Selective probiotic bacteria induce IL-10-producing regulatory $\mathrm{T}$ cells in vitro by modulating dendritic cell function through dendritic cell-specific intercellular adhesion molecule 3-grabbing nonintegrin. J Allergy Clin Immunol 115(6):1260-1267. https://doi.org/10.1016/j.jaci.2005.03.036

47. Schulke S (2018) Induction of interleukin-10 producing dendritic cells as a tool to suppress allergen-specific T helper 2 responses. Front Immunol 9:455. https://doi.org/10.3389/fimmu.2018.00455

48. Bacher P, Heinrich F, Stervbo U, Nienen M, Vahldieck M, Iwert C, Vogt K, Kollet J, Babel N, Sawitzki B, Schwarz C, Bereswill S, Heimesaat MM, Heine G, Gadermaier G, Asam C, Assenmacher M, Kniemeyer O, Brakhage AA, Ferreira F, Wallner M, Worm M, Scheffold A (2016) Regulatory T cell specificity directs tolerance versus allergy against aeroantigens in humans. Cell 167(4):10671078 e16. https://doi.org/10.1016/j.cell.2016.09.050

49. Ashjaei K, Bublin M, Smole U, Lengger N, Hafner C, Breiteneder H, Wagner S, Hoffmann-Sommergruber K (2015) Differential T-helper cell polarization after allergen-specific stimulation of autologous dendritic cells in polysensitized allergic patients. Int Arch Allergy Immunol 166(2):97-106. https://doi.org/10.1159/ 000375405

50. Mabbott NA, Donaldson DS, Ohno H, Williams IR, Mahajan A (2013) Microfold (M) cells: important immunosurveillance posts in the intestinal epithelium. Mucosal Immunol 6(4):666-677. https://doi.org/10.1038/mi.2013.30

51. Stagg AJ (2018) Intestinal dendritic cells in health and gut inflammation. Front Immunol 9:2883. https://doi.org/10.3389/fimmu. 2018.02883

Publisher's Note Springer Nature remains neutral with regard to jurisdictional claims in published maps and institutional affiliations. 